\title{
A RELAÇÃO ENTRE TUBERCULOSE PULMONAR E COVID-19: UMA REVISÃO BIBLIOGRÁFICA.
}

Categoria: Saúde coletiva/Epidemiologia.

Instituição: Centro Universitário São Camilo

Autores: Crotti, G.P; Bassoto, A.F; Whitaker, B.S; Santos, R.R; Ikeda, R.K.

Orientadora: Barcelos, D.

Dados do autor principal:

Endereço: R. Salvador Frezolone, 89, SBC, SP.

Telefone: (11) 966355392

Email: giuliana.crotti060@gmail.com 


\section{A RELAÇÃO ENTRE TUBERCULOSE PULMONAR E COVID-19: UMA REVISÃO} BIBLIOGRÁFICA.

Categoria: Saúde coletiva/Epidemiologia.

Descritores: Pulmonary Tuberculosis, Covid-19, Coinfection. 


\section{A RELAÇÃO ENTRE TUBERCULOSE PULMONAR E COVID-19: UMA REVISÃO BIBLIOGRÁFICA.}

Autores: Crotti, G.P; Bassoto, A.F; Whitaker, B.S; Santos, R.R; Ikeda, R.K.

Orientadora: Barcelos, D

Email: giuliana.crotti060@gmail.com

Instituição: Centro Universitário São Camilo

INTRODUÇÃO: A TB é uma das maiores causas de mortalidade no mundo e apesar de redução na sua incidência, permanece um fardo para a saúde pública. A coinfecção Tuberculose (TB)/Coronavírus (CV) pode amplificar uma à outra. Ambas possuem os mesmos sintomas, compartilham fatores de risco e podem induzir profissionais de saúde a negligenciarem a TB como diagnóstico diferencial. METODOLOGIA: Trata-se de uma revisão bibliográfica realizada na base de dados PubMed, com os descritores "Pulmonary Tuberculosis", "Covid-19" e "Coinfection" unidos pelo operador booleano AND. Foram selecionados 18 artigos após critérios de inclusão e exclusão. OBJETIVOS: Esse trabalho objetiva identificar possíveis relações entre a Tuberculose Pulmonar e Covid-19. RESULTADOS: Pacientes com TB são vulneráveis e apresentam maior letalidade em coinfecção. Contudo, a resposta inflamatória de ambas é distinta, enquanto a TB evita a superestimulação do sistema imunológico, o CV apresenta uma resolução mais agressiva. Ambas estimulam as mesmas citocinas inflamatórias e a somatória das repostas imunes contra elas leva ao acúmulo exacerbado de células inflamatórias pulmonares, à necrose e promove uma tempestade de citocinas. Indivíduos com história prévia de TB apresentam cicatrizes da fibrose pulmonar, que tornam as vias aéreas propensas à infecção por $\mathrm{CV}$, além de possibilidade de pior prognóstico. A TB ativa aumenta a probabilidade de COVID-19 grave devido à grande quantidade de células imunes encontradas nos pulmões. Outro dado é que a utilização de imunossupressores deve ser feita com cautela em paciente graves com $\mathrm{CV}$, pois compromete a intensidade da resposta imune. Em indivíduos já coinfectados, a resposta deficiente compromete a integridade do granuloma, favorecendo a reativação. $\mathrm{O}$ uso por pessoas infectadas apenas pelo $\mathrm{CV}$ facilita a infecção por patógenos oportunistas, semelhante à TB. Os dados sobre a letalidade da coinfecção não são concretos, entretanto, em estudos prévios, a coinfecção foi controlável e a letalidade variou entre 5\% a $11,6 \%$. Outros autores descreveram que, 53\% dos pacientes que apresentaram CV, previamente foram diagnosticados com TB, em $18 \%$ a infecção era simultânea e a grande maioria apresentava TB ativa. CONCLUSÃO: A associação TB e CV deve ser observada atentamente, visto o conhecimento prévio de coinfecção entre essas condições e além de que mais estudos nesta área são necessários. DESCRITORES: Pulmonary Tuberculosis, Covid-19, Coinfection. 


\section{A RELAÇÃO ENTRE TUBERCULOSE PULMONAR E COVID-19: UMA REVISÃO BIBLIOGRÁFICA.}

\section{INTRODUÇÃO}

A Tuberculose (TB) é uma das doenças mais antigas conhecidas pela humanidade, tendo surgido há aproximadamente 150 milhões de anos. Trata-se de uma doença infecciosa causada pelo Mycobacterium tuberculosis (Mtb) que compromete principalmente os pulmões e cuja principal forma de manifestação clínica é a TB pulmonar (TBP). Com uma prevalência mundial de aproximadamente 1,7 bilhões de pessoas contaminadas, é considerada uma das maiores causas de mortalidade no mundo, e apesar da redução na sua incidência, permanece uma importante questão de saúde pública. $(1,2,3,10)$

Em contrapartida ao grande período de contato entre a espécie humana e o Mtb, o SARSCoV-2 foi notificado em dezembro de 2019, inicialmente na Cidade de Wuhan, na China, na forma de pneumonia inexplicada. Alastrou-se rapidamente pelos continentes, levando ao reconhecimento pela OMS da pandemia pelo COVID-19 em 11 de março de 2020, e é considerada uma emergência de saúde pública pela sua capacidade de sobrecarregar os sistemas de saúde. $(8,19)$

Apesar de suas particularidades evidentes, as doenças compartilham semelhanças clínicas e epidemiológicas como sintomas de febre, tosse, falta de ar e fraqueza, sendo que a história clínica da TBP é cronificada em relação a COVID-19. Além disso, uma maior mortalidade relacionada a casos de associação entre infecções virais e bacterianas já foi descrito na literatura e observado em pacientes portadores da coinfecção TBP/Influenza, o que elucida a relevância dos conhecimentos acerca da relação TB-COVID. $(8,13)$

\section{OBJETIVO}

Por se tratarem de doenças respiratórias cujos quadros clínicos assemelham-se e dado o impacto de ambas as doenças na saúde pública, suas similaridades e a maior mortalidade de uma associação entre infecções virais e bacterianas, objetivamos comparar suas principais características e manifestações a fim de identificar possíveis relações entre a Tuberculose Pulmonar e COVID-19, buscando expandir os conhecimentos acerca da coinfecção TB-COVID. 


\section{METODOLOGIA}

O trabalho trata-se de uma revisão bibliográfica de artigos científicos sobre o tema “Tuberculose pulmonar e Covid-19”. A base de dados utilizada foi MedLine via PubMed por meio de sítios online, nos quais foram efetuadas as buscas com os seguintes descritores: "Pulmonary Tuberculosis", “Covid-19” e "Coinfection”, utilizando-se do operador booleano AND.

Foram encontrados 40 artigos no período de 2020 a 2021, posteriormente foram adicionados os filtros "English", "Portuguese" e "Humans" sendo que, após isso, foram obtidos 31 artigos dos quais analisaram-se o título, resumo e conteúdo, restando 18 estudos para leitura e confecção do trabalho. Excluíram-se 7 artigos pelo título, 2 pela não disponibilidade de forma gratuita e, por fim, 4 onde o assunto não era pertinente ao conteúdo da revisão. Assim, teve-se como resultado 18 artigos finais para a realização da discussão.

DESCRITORES: "Pulmonary Tuberculosis", "Covid-19” e "Coinfection"

Aplicação dos filtros:

"English", "Portuguese"

e "Coinfection"

\section{PubMed: 40 artigos}

\section{1 artigos}




\section{DISCUSSÃO}

\section{SÍNDROMES RESPIRATÓRIAS}

A coinfecção de Tuberculose (TB) e outros Coronavírus como SARS-COV-1 e MERS-COV foi previamente observada durante os surtos de 2003 e 2012, respectivamente. Sobre tal associação foi descrito que ambas as doenças poderiam ampliar uma à outra, com redução da imunidade e consequente infecção aguda ou reativação de uma infecção latente (é considerado que aproximadamente $25 \%$ da população mundial é portadora da infecção latente). Tem-se como pressuposto que pacientes desenvolvem a forma ativa de TB pulmonar após serem infectados pelo novo Coronavírus, tal processo decorre de uma supressão transitória da imunidade celular induzida pelo SARS-COV-2. Dessa forma, agravando ou predispondo uma nova infecção por Mycobacterium tuberculosis (MTB). Por fim, a associação Tuberculose e SARS-COV-2 deve ser observada atentamente, visto o conhecimento prévio de coinfecção entre essas condições. (7-12)

\section{RELEVÂNCIA DA TB}

A tuberculose é uma das dez maiores causas de mortalidade pelo mundo, e ainda que apresente uma leve redução na sua incidência, permanece um fardo para as autoridades responsáveis pelas políticas de saúde pública. O seu diagnóstico padrão ouro é pela cultura do bacilo e testagem da susceptibilidade antimicrobiana, porém pela limitação de acesso aos laboratórios de microbiologia, dificulta-se o diagnóstico precoce, que previne a disseminação da doença. É importante ressaltar que existem formas fármaco-resistentes da MTB, em que se pode esperar uma maior mortalidade, inclusive de pacientes jovens (em 2018, estima-se que quase metade da carga global de 10 milhões de doentes possuíam a forma resistente). $(8-10,13,14)$

\section{SEMELHANÇAS E DIFERENÇAS}

Tanto a infecção por TB quanto a infecção por COVID costumam acarretar manifestações respiratórias, principalmente a tosse e dispneia. TB e COVID também podem levar a episódios de febre e astenia. A semelhança na sua sintomatologia e o atual foco no COVID pode induzir profissionais de saúde a negligenciar a tuberculose como um possível diagnóstico diferencial. Apesar de casos de coinfecção ainda serem escassos na literatura, essa possibilidade já foi relatada por alguns autores. Dessa forma, levando em conta que pacientes infectados por TB com idade avançada ou imunossupressão ou comprometimento respiratório são mais suscetíveis a casos graves de COVID, exames como baciloscopia e RT-PCR são fundamentais para determinar o diagnóstico correto do paciente o mais rápido possível e evitar coinfecção. Sobre os fatores de risco para ambas as infecções, temos: sexo (homens), hábitos de vida (tabagismo, etilismo), doenças (diabetes, obesidade, 
portadores de HIV, doença renal, hepática ou cardiovascular) e determinantes sociais (áreas superlotadas, população pobre e desnutrida). (7,9,10,13-16,19)

Tabela 1 - Comparação entre aspectos principais da TBP e COVID-19

\begin{tabular}{|c|c|c|}
\hline Característica & TBP & COVID-19 \\
\hline Modo de transmissão & - Gotículas de Mtb. & - Gotículas de SARS-CoV-2. \\
\hline Quadro clínico & $\begin{array}{l}\text { - Tosse crônica com mais de } 3 \\
\text { semanas, associada a } \\
\text { expectoração mucoide ou } \\
\text { hemoptoica. } \\
\text { - Dispneia. } \\
\text { - Febre e suores noturnos. } \\
\text { - Calafrios. } \\
\text { - Perda ponderal e anorexia. } \\
\text { - Fadiga. }\end{array}$ & $\begin{array}{l}\text { - Tosse seca. } \\
\text { - Dispneia. } \\
\text { - Febre. } \\
\text { - Calafrios. } \\
\text { - Náusea, vômito e diarreia. } \\
\text { - Fadiga. } \\
\text { - Cefaleia, mialgia e dor de } \\
\text { garganta. } \\
\text { - Anosmia e agnosia. } \\
\text { - Congestão nasal e nariz } \\
\text { escorrendo. }\end{array}$ \\
\hline Diagnóstico & $\begin{array}{l}\text { - Teste rápido molecular para } \\
\text { Mtb. } \\
\text { - Radiografia de tórax. } \\
\text { - Baciloscopia direta. } \\
\text { - Cultura. } \\
\text { - Teste de sensibilidade. } \\
\text { - Tomografia. } \\
\text { - Biopsia. }\end{array}$ & $\begin{array}{l}\text { - Radiografia de tórax. } \\
\text { - Tomografia. } \\
\text { - Teste rápido molecular para } \\
\text { SARS-CoV-2. }\end{array}$ \\
\hline Tratamento & $\begin{array}{l}\text { - Disponibilizado pelo SUS. } \\
\text { - Rifampicina, Isoniazida, } \\
\text { Pirazinamida e Etambutol. } \\
\text { - } 6 \text { meses a } 2 \text { anos. } \\
\text { - Tratamento diretamente } \\
\text { observado. }\end{array}$ & - Sem tratamento definido. \\
\hline
\end{tabular}

4. MEDIDAS DE PREVENÇÃO 
É de suma importância a utilização de máscaras e EPIS nos serviços de saúde, pois sabe-se que o vírus da Sars-Cov-2 é disseminado através de gotículas. Pacientes de TB são especialmente vulneráveis, como mencionado anteriormente, aumentando ainda mais a letalidade no caso de uma possível coinfecção. Devido a necessidade de isolamento social, casos de tuberculose acabam sendo subnotificados, levando a aumento de casos não diagnosticados. Além disso, o isolamento social levou à necessidade de automedicação dos pacientes, para evitar contágio por COVID-19 e preservar os doentes imunodeprimidos. Contudo, o distanciamento social pode aumentar o preconceito relacionado a comunidades marginalizadas, desencorajando essas de buscar atendimento médico e aumentando a gravidade da tuberculose. A telemedicina foi essencial para o tratamento continuado de tuberculose e monitorização das condições de pacientes, levantando a necessidade de adaptação dos profissionais a um ambiente digital, além do redirecionamento do fluxo de medicamentos para farmácias ao invés de hospitais, para evitar exposição desnecessária de cada paciente. (8-11,17-20)

\section{FISIOPATOLOGIA}

A resposta inflamatória em ambas as doenças é distinta: enquanto o MTB busca evitar a superestimulação do sistema imunológico, ocasionando uma infecção crônica associada a formação de granuloma e necrobiose, o SARS-CoV-2 apresenta uma resolução mais agressiva, acarretando piroptose. Em uma coinfecção, a MTB e o SARS-CoV-2 podem atuar de forma sinérgica apesar de suas respostas divergentes. $(7,8,21)$

O MTB interfere no microambiente e na arquitetura pulmonar. Durante a fase latente da infecção, as citocinas TNF e INF-gama representam um importante papel na indução da resposta inflamatória crônica e na manutenção da estrutura do granuloma. Estas mesmas citocinas desempenham função igualmente fundamental na resposta inflamatória contra o SARS-CoV-2. A somatória das repostas imunes contra ambos os microrganismos leva ao acúmulo exacerbado de células inflamatórias no pulmão, à morte celular por necrose e piroptose e ao aumento na dispersão de DAMPs, potencializando o feedback inflamatório nas vias aéreas e promovendo uma tempestade de citocinas e posterior imunopatologia. $(7,8,21)$

Durante o período de latência o microrganismo continua a se multiplicar causando o surgimento de lesões cavitarias. Tais lesões reduzem a superfície disponível para a troca gasosa, a bronquiectasia decorrente restringe o fluxo de ar e a obstrução dos capilares compromete a drenagem do fluido pulmonar. Essas alterações afetam o funcionamento e a defesa do trato respiratório tornando-o mais suscetível a complicações graves. Indivíduos com história prévia de TB apresentam cicatrizes subsequentes a fibrose do tecido pulmonar que tornam as vias aéreas inferiores mais propensas a infecção por SARS-CoV-2, além de apresentar maior chance de pior prognóstico. $(7,8,21)$ 
O dano provocado aos pulmões resultante da TB somado ao impacto promovido pelas duas infecções na imunidade local são consideradas como a principal razão para o maior risco de infecção por patógenos transmitidos pelo ar, como é o caso do SARS-CoV-2.(7,8,21)

Outros fatores como o status da TB e a presença ou ausência de uma resposta imune adaptativa específica para SARS-CoV-2 também podem desempenhar um papel no desenvolvimento e na exacerbação da COVID-19 na população coinfectada. A TB ativa aumenta o risco de COVID-19 grave devido à grande quantidade de células do sistema imune encontradas nos pulmões, o que, somado à tempestade de citocinas e à imunopatologia, eleva a possibilidade de evolução da doença para quadros graves ou fatais. $(7,8,19)$

A presença de células TCD4+ específicas para o antígeno SARS-CoV-2 juntamente com uma resposta coordenada e ampla com as células TCD8+e B apresenta aspecto protetor em pacientes em quadro ativo de COVID-19 e está relacionada a menor gravidade da doença. Quando ativados, os linfócitos TCD4+ maturam-se em células T helper 1 (Th1) patogênicas que atuam sobre o meio criando um ambiente de citocinas favorável à maturação de monócitos com alta expressão de IL-6, o que acelera a inflamação. Em contrapartida, em pacientes com quadro grave da infecção, foi relatado a presença de superativação de células TCD4+, maturadas em células Th17 em detrimento das células Th1, o que, somado à ação das células TCD8+, acarreta alta citotoxicidade no sangue periférico. $(7,8,19)$

A utilização de medicamentos imunossupressores, como os glicocorticoides, deve ser feita com cautela em paciente graves com COVID-19, pois compromete a intensidade da resposta imune. Em indivíduos já coinfectados, a resposta imune deficiente compromete a integridade do granuloma, favorecendo a reativação da TB. O uso por pessoas infectadas apenas pelo SARS-CoV-2 facilita a infecção por patógenos oportunistas, como é o caso da TB. $(7,8,19,21)$

\section{EPIDEMIOLOGIA}

Os dados sobre a letalidade da coinfecção TB e Covid-19 não são estabelecidos de forma concreta, mas existem estudos que aproximam seu valor. Um estudo italiano observou 20 pacientes apresentando a combinação das duas doenças, sendo que a associação geralmente foi controlável e a letalidade foi de 5\%. Em uma outra análise feita por Motta et al. de dois coortes de 49 e 20 pacientes a taxa de letalidade foi de $11,6 \%$. Tadolini et. al descreveram que dos 49 pacientes, $53 \%$ apresentavam Tuberculose previamente ao diagnóstico de Covid-19, 18\% a infecção era simultânea e a grande maioria apresentava Tuberculose ativa. Ademais, em um estudo observacional retrospectivo, com um 
total de 1.073 pacientes sendo que 22 casos apresentavam coinfecção TB e Covid-19 a taxa de mortalidade de $27,3 \%$ foi maior que nos estudos anteriormente descritos. $(22,23)$ 


\section{CONCLUSÃO:}

A coinfecção Tuberculose Pulmonar e COVID-19 deve ser de conhecimento por parte dos profissionais na abordagem ao paciente com quadros respiratórios, tendo em vista que a associação entre a TB e outros Coronavírus e a possível ação sinérgica envolvendo essas doenças podem levar a quadros de maior gravidade e pior prognóstico. Estudos adicionais laboratoriais e de campo podem contribuir para uma maior compreensão e detalhamento sobre tal relação e para um melhor suporte à prática clínica. 


\section{REFERÊNCIAS}

1. Veronesi R FR. Tratado de Infectologia. 4th ed. Rio de Janeiro: Atheneu; 2010.

2. M. C, Tavares VR, Campos DJ, Silva AC, Bueno SK. Tuberculose: Uma Revisão De Literatura. Rev Eletrônica da Fac Ceres. 2013;2(1):34-47.

3. Mohajan HK. Tuberculosis is a Fatal Disease among Some Developing Countries of the World. Am J Infect Dis Microbiol [Internet]. 2015;3(1):18-31. Available from: http://pubs.sciepub.com/ajidm/3/1/4/index.html

4. Braunwald, E, Kasper DL, Fauci AS, Jameson JL, Longo DL HS. Medicina Interna de Harrison. 19th ed. Porto Alegre: AMGH; 2017.

5. Ahmad S. Pathogenesis, immunology, and diagnosis of latent mycobacterium tuberculosis infection. Clin Dev Immunol. 2011;2011.

6. Secrearia Estadual de Saúde do Paraná. Tuberculose - Secretaria da Saúde [Internet]. 2019. Available from: http://www.saude.pr.gov.br/modules/conteudo/conteudo.php?conteudo=939

7. Orozco JAM, Tinajero ÁS, Vargas EB, Cueva AID, Escobar HR, Alcocer EV, et al. COVID-19 and tuberculosis coinfection in a 51-year-old taxi driver in Mexico city. Am J Case Rep. 2020;21:1-6.

8. Singh A, Prasad R, Gupta A, Das K, Gupta N. Severe acute respiratory syndrome coronavirus- 2 and pulmonary tuberculosis: convergence can be fatal. Monaldi Arch Chest Dis. 2020;9(3):441-50.

9. Tadolini M, Codecasa LR, García-García JM, Blanc FX, Borisov S, Alffenaar JW, et al. Active tuberculosis, sequelae and COVID-19 co-infection: First cohort of 49 cases. Eur Respir J. 2020;56(1).

10. Bandyopadhyay A, Palepu S, Bandyopadhyay K, Handu S. COVID-19 and tuberculosis coinfection: A neglected paradigm. Monaldi Arch Chest Dis. 2020;90(3):518-22.

11. Sarinoglu RC, Sili U, Eryuksel E, Yildizeli SO, Cimsit C, Yagci AK. Tuberculosis and COVID-19: An overlapping situation during pandemic. J Infect Dev Ctries. 2020;14(7):721-5.

12. Zumla A, Marais BJ, McHugh TD, Maeurer M, Zumla A, Kapata N, et al. COVID-19 and tuberculosis-threats and opportunities. Int J Tuberc Lung Dis. 2020;24(8):757-60.

13. Crisan-Dabija R, Grigorescu C, Pavel CA, Artene B, Popa IV, Cernomaz A, et al. Tuberculosis and COVID-19: Lessons from the Past Viral Outbreaks and Possible Future Outcomes. Can Respir J. 2020;2020. 
14. Chen ZYJ, Wang Q, Liu W, Nan QZJ, Huang H, Wu Y, et al. Three Patients with COVID19 and Pulmonary Tuberculosis, Wuhan, China, January-February 2020. Emerg Infect Dis. 2020;26(11):2754-7.

15. Motta I, Centis R, D’Ambrosio L, García-García JM, Goletti D, Gualano G, et al. Tuberculosis, COVID-19 and migrants: Preliminary analysis of deaths occurring in 69 patients from two cohorts. Pulmonology. 2020;26(4):233-40.

16. Khurana AK, Aggarwal D. The (in)significance of TB and COVID-19 co-infection. Eur Respir J [Internet]. 2020;56(2):1-4. Available from:

http://dx.doi.org/10.1183/00000000.00000000

17. Behera D. TB control in India in the COVID era. Indian J Tuberc. 2021;68(1):128-33.

18. Watts K, McKeown A, Denholm J, Baker AM. Responding to COVID-19: Adjusting TB services in a low-burden setting. Int J Tuberc Lung Dis. 2020;24(8):866-9.

19. Visca D, Ong CWM, Tiberi S, Centis R, D'Ambrosio L, Chen B, et al. Tuberculosis and COVID-19 interaction: A review of biological, clinical and public health effects.

Pulmonology [Internet]. 2021;27(2):151-65. Available from:

https://doi.org/10.1016/j.pulmoe.2020.12.012

20. Yadav S, Rawal G. The case of pulmonary tuberculosis with covid-19 in an indian male-a first of its type case ever reported from south asia. Pan Afr Med J. 2020;36(374):1-5.

21. Mousquer GT, Peres A, Fiegenbaum M. Pathology of TB/COVID-19 Co-Infection: The phantom menace. Tuberculosis. 2021;126(September 2020):17-22.

22. Stochino C, Villa S, Zucchi P, Parravicini P, Gori A, Raviglione MC. Clinical characteristics of COVID-19 and active tuberculosis co-infection in an Italian reference hospital. Eur Respir J. 2020;56(1).

23. Gupta N, Ish P, Gupta A, Malhotra N, Caminero JA, Singla R, et al. A profile of a retrospective cohort of 22 patients with COVID-19 and active/treated tuberculosis. Eur Respir J. 2020;56(5):3-7. 